\title{
Alternate Designs of Ultrasonic Absorptive Coatings for Hypersonic Boundary Layer Control
}

\author{
Guillaume A. Brès, ${ }^{*}$ Matthew Inkman, ${ }^{\dagger}$ and Tim Colonius ${ }^{\ddagger}$ \\ California Institute of Technology, Pasadena, CA 91125, U.S.A. \\ Alexander V. Fedorov ${ }^{\S}$ \\ Moscow Institute of Physics and Technology, Zhukovsky, Moscow region, 140180, Russia
}

\begin{abstract}
Numerical simulations of the linear and nonlinear two-dimensional Navier-Stokes equations are used to parametrically investigate hypersonic boundary layers over ultrasonic absorptive coatings consisting of a uniform array of rectangular pores (slots) with a range of porosities and pore aspect ratios. Based on our previous work, we employ a temporally evolving approximation appropriate to slowly-growing second-mode instabilities. We consider coatings operating in attenuative regimes where the pores are relatively deep and acoustic waves and second mode instabilities are attenuated by viscous effects inside the pores, as well as cancellation/reinforcement regimes with alternating regions of local minima and maxima of the coating acoustic absorption, depending on the frequency of the acoustic waves. The focus is on reinforcement cases which represent a worst case scenario (minimal second-mode damping). For all but one of the cases considered, the linear simulations confirm the results of linear instability theory that employs an approximate porous-wall boundary condition. A particular case with a relatively shallow cavities and very high porosity showed the existence of a shorter wavelength instability that is not predicted by theory. Finally, nonlinear simulations of the same cases led to the same conclusions as linear analysis; in particular, we did not observe any "tripping" of the boundary layer by small scale disturbances associated with individual pores.
\end{abstract}

\section{Nomenclature}

Ar Cavity aspect ratio, $2 b / H$

a Speed of sound

$b \quad$ Cavity half-width

$f \quad$ Frequency, $f^{*} H / a_{w}$

$H \quad$ Cavity depth

$M_{e} \quad$ Mach number, $U_{e} / a_{e}$

$N_{\text {pore }}$ Number of pores per $\Lambda$

$\mathcal{R} \quad$ Reflection coefficient

$R e \quad$ Acoustic Reynolds number, $\rho_{w} a_{w} b / \mu_{w}$

$R e_{\delta} \quad$ Reynolds number, $\rho_{e} U_{e} \delta / \mu_{e}$

$s \quad$ Cavity spacing

$T$ Temperature

$t \quad$ Time

$U \quad$ Freestream streamwise velocity

$u, v \quad$ Streamwise and normal velocities

$x, y \quad$ Streamwise and normal directions

$\delta \quad$ Boundary layer thickness $\theta \quad$ Angle of incidence of acoustic wave

$\Lambda \quad$ Streamwise extent of the domain

$\lambda \quad$ Streamwise wavelength

$\mu \quad$ Dynamic viscosity

$\nu \quad$ Kinematic viscosity

$\rho \quad$ Density

$\sigma \quad$ Temporal growth rate of second mode, $\sigma^{*} \delta / U_{e}$

$\phi \quad$ Porosity, $2 b / s$

$\omega \quad$ Angular frequency of second mode, $\omega^{*} \delta / U_{e}$

Superscript

' Linear pertubation quantity

* Dimensional quantity

Subscript

$0 \quad$ Stagnation quantity

e $\quad$ Property at the boundary layer edge

$w \quad$ Property at the wall

* Senior Aeroacoustic Engineer, current address: Exa Corporation, Brisbane, CA 94005; Member AIAA

$\dagger$ Graduate student, Dept. of Mechanical Engineering

$\ddagger$ Professor, Dept. of Mechanical Engineering; Member AIAA

$\S$ Professor, Dept. of Aeromechanics and Flight Engineering; Member AIAA 


\section{Introduction}

Laminar-turbulent transition during hypersonic flight represents a severe restriction to vehicle performance, ${ }^{1}$ since early transition causes a significant increase (by a factor of 3-8) in heat transfer that translates to higher cost and weight of thermal protection systems (TPS). ${ }^{2,3}$ State-of- the-art active and reactive laminar flow control (LFC) methods ${ }^{4}$ seem to be not practical under the severe conditions of hypersonic flight. In contrast, a passive ultrasonic absorptive coating (UAC), which consists of a thin perforated layer of regular microstructure, has been shown to significantly increase laminar run in wind-tunnel experiments. ${ }^{5,6}$ Additional experimental and theoretical studies ${ }^{7-10}$ have confirmed the UAC stabilizing effect on the second mode (or Mack mode), which is the dominant instability causing transition in predominantly two-dimensional boundary layers at hypersonic speed (rather than the first mode associated with Tollmien-Schlichting waves). These studies showed the robustness of the UAC stabilization concept and motivated our current direct numerical simulations (DNS) and theoretical efforts to mature the UAC-LFC methodology and provide a basis for design of UAC-TPS test articles that could be manufactured and deployed on a flight vehicle.

In previous works, two unit problems that bracket the flow physics of the full UAC problem were investigated. The first study ${ }^{11}$ introduced a computationally tractable and efficient method to account for the detailed flow physics associated with micro-cavities that attenuate second-mode instabilities in hypersonic boundary layers. Linear stability theory ${ }^{5}$ (LST) and DNS provided evidence that it is sufficient for many purposes to consider temporally (rather than spatially) evolving boundary layers and instabilities. As the computational domain can be limited to a single wavelength of the instability, the computational cost is significantly reduced. The same approach has been used in a recent work on UAC with streamwise slots and square pores, with similar results. ${ }^{12}$

The second study ${ }^{13,14}$ examined the interaction of acoustic waves with an array of equally-spaced twodimensional micro-cavities on a flat plate without external boundary-layer flow. This fundamental problem is of interest here because the second-mode instability represents high-frequency (ultrasonic) acoustic waves trapped in the boundary layer. In particular, the DNS and theoretical modeling ${ }^{15}$ highlighted, for sufficiently shallow cavities, the presence of alterning local minima and maxima of the reflection coefficient (i.e., maxima and minima of the absorptive acoustic property of the coating) at some specific frequencies. This behavior is due to reflections of acoustic waves from the bottom of the cavities that lead to destructive/constructive reinforcement at these specific frequencies. Such properties are mainly observed for pores of large aspect ratio, because for cavities that are deep and narrow, the acoustic waves are strongly attenuated by viscous effects in the pores.

In practice, the second-mode frequency is related to the boundary layer thickness and decreases as the instability travels downstream in spatially-evolving boundary layers. In contrast, the acoustic properties of the coating are independent of the boundary layer properties. Consequently, it is expected that the operating condition of a uniform UAC would alternate between local minima and maxima of the coating acoustic absorption, depending on the streamwise position along the vehicle. The conservative way to design coatings is therefore to pick the attenuative regime since the performance will not vary much along the streamwise position on the vehicle, whereas the cancellation/reinforcement regime leads to significant fluctuation of the performance. However, an optimum attenuative coating typically requires many deep pores, which could be a difficult to manufacture.

In this paper, the temporal stability analysis is extended to investigate alternatives to the conservative UAC designs, under the assumption that their stabilizing perfomances are directly related to their acoustic scattering performances. Nine different coatings are considered, spanning the range of typical UAC parameters relevant to practical applications. The properties of the second-mode instability for a hypersonic boundary layer over a flat plate and over the different UAC are compared, using linear stability theory ${ }^{5}$ (LST) and linear direct numerical simulations (LDNS). First, the flow conditions are set up such that the frequency of the dominant second-mode instability matches a frequency of minimum absorption of the UAC, as identified by the acoustic scattering study. This particular setup corresponds to a reinforcement regime (i.e., "worst-case scenario"), to quantify the performance of the different UAC in unfavorable conditions. Then, a second configuration where the UAC operate at a cancellation regime with maximum acoustic absorption is also considered (i.e., "best-case scenario"), to confirm that stronger stabilization is obtained in this case. Finally, nonlinear direct numerical simulations of the UAC including the outer flow are performed to validate these alternate UAC designs, and examine potential detrimental and tripping effects. 


\section{Acoustic properties of porous coatings}

\section{A. Previous work}

The acoustic scattering properties of two-dimensional porous coatings were investigated using DNS and theoretical modeling. ${ }^{13-15}$ The existing theoretical model considered the reflection of plane monochromatic acoustic waves of an ultrasonic frequency band from a plane surface covered by a porous coating (figure 1). The reflection coefficient, $\mathcal{R}$, characterizing the ratio of the reflected wave amplitude to the incident wave amplitude, is computed as a function of the acoustic wave frequency $f=f^{*} H / a_{w}$ and angle of incidence $\theta$, for coatings of different porosity $\phi=2 b / s$, at various acoustic Reynolds number $R e=\rho_{w} a_{w} b / \mu_{w}$ relevant to hypersonic flight. Here, $b$ is the cavity half-width, $s$ is the pore spacing, $H$ is the pore depth, $a$ is the speed of sound, $\rho$ and $\mu$ are the gas density and viscosity, respectively. The subscript $w$ denotes the property at the wall, and the superscript $*$ refers to the dimensional quantity.

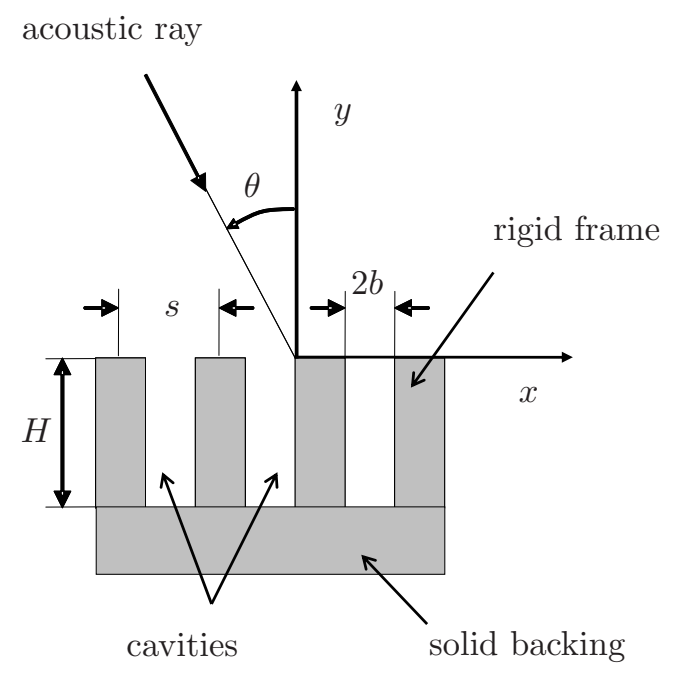

Figure 1. Schematic of the reflection of acoustic wave from equally spaced 2-D cavities.

The key assumptions and main results are summarized here, since they are the starting point of the present work:

1. The theoretical model assumes that the cavity half-width $b$, depth $H$ and spacing $s$ satisfy the conditions $b \sim s \ll \lambda_{a c s}$, and $H \sim \lambda_{a c s}$, where $\lambda_{a c s}$ is the wavelength of incident acoustic wave. These conditions can be reformulated using the porosity $\phi$, the cavity aspect ratio $A r=2 b / H$ and the nondimensionalized frequency of the incident acoustic wave $f$ as

$$
\frac{1}{\phi} \operatorname{Arf} \ll 1, \quad \quad \frac{1}{2} \operatorname{Arf} \ll 1, \quad f \sim 1 .
$$

For typical UAC parameters, the range of frequencies $0.1 \leq f \leq 1.5$ corresponds to the ultrasonic frequency band, and is sufficient to capture the frequency of the most amplified second-mode instability waves observed in experiments ${ }^{6,16}$ and numerical simulations. ${ }^{5,11,17}$

2. The reflection coefficient is largely independent of the angle of incidence, up to $\theta \approx 30^{\circ}$ for all the conditions considered. For the second-mode instability waves, the angle of incidence was shown to be smaller than $23^{\circ}$ for cases relevant to UAC design. ${ }^{18}$ Therefore, our analysis will only focus on the acoustic properties of the porous coating at normal incidence (i.e., $\theta=0^{\circ}$ ).

3. There is a critical acoustic Reynolds number $R e_{\text {crit }}$ for the cancellation/reinforcement regime, above which acoustic disturbances are not completely absorbed inside the pores. In that case, interference between incoming and outgoing (reflected from the cavity bottom) waves leads to a significant decrease of the reflection coefficient at some specific frequencies. In the appendix, this critical Reynolds number 
is characterized and the presence of reflection from the cavity bottom can be anticipated for a range of acoustic Reynolds numbers $50 \leq R e \leq 1000$ relevant for practical UAC in high-altitude hypersonic flight.

4. A simple model for the prediction of these specific frequencies was introduced and led to estimates in good agreement with the theoretical and numerical results: $f \approx 0.25,0.75$, etc. These frequencies, corresponding to local minima of the reflection coefficient, are largely independent of the Reynolds number.

5. The DNS showed the presence of a resonant acoustic mode caused by the coupling of small-scale scattered waves generated by neighboring pores near the UAC surface. In this mechanism, the resonant frequency can be estimated by the ratio of the porosity by the cavity aspect ratio, i.e., $f_{r e s}=\phi / A r$. In most cases, these frequencies are higher than the ultrasonic frequency band relevant for UAC. Nevertheless, the resonant acoustic modes may trip the outer boundary-layer flow similar to small-scale distributed roughness.

\section{B. Parametric study}

In our previous studies, the 2-D micro-cavities considered had a constant length to depth ratio $A r=0.12$, matching the aspect ratio of the cylindrical cavities used in the experiment by Rasheed et al.. ${ }^{6}$ To further investigate the effect of the cavity depth and the porosity, additional simulations are performed. Typically, coatings with very deep pores $(A r<0.05)$ or very high porosity $(\phi>0.8)$ are not of interest for practical applications. Therefore, nine different coatings are considered here, for aspect ratio $A r=0.06,0.12,0.3$, and porosity $\phi=0.2,0.48,0.8$. These parameters span the range of typical coating designs used in most experimental and theroretical studies on UAC.

The reflection coefficient obtained from DNS is compared with the theoretical modeling in figure 2, for the nine different designs. Overall, the comparison shows again excellent agreement between the two methods, especially for low aspect ratio and low frequency. The discrepancies at $A r=0.3$ are due to the assumptions in the theory mentioned in the previous section. Nevertheless, the theoretical model ${ }^{15}$ is used in the rest of the paper to efficiently provide estimates of the acoustic properties of the different coatings considered.

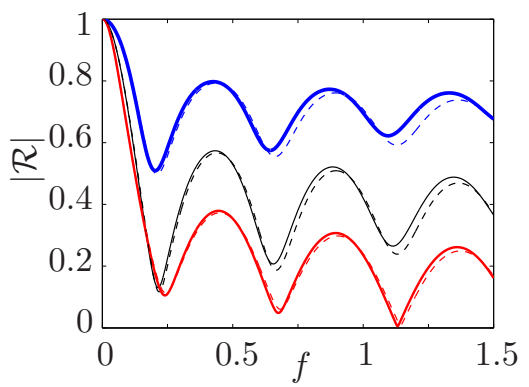

$(a)$

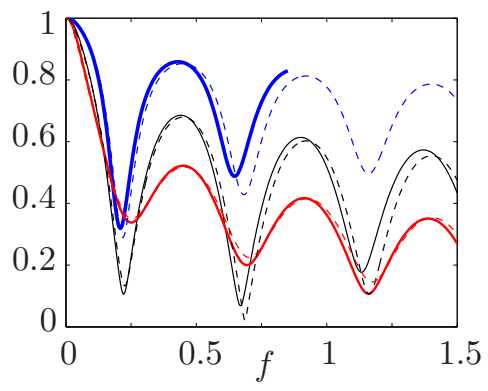

(b)

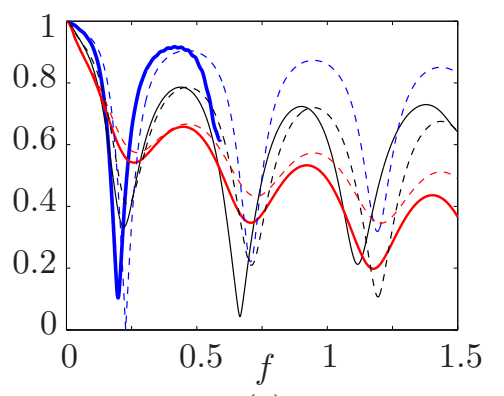

$(c)$

Figure 2. Comparison of the reflection coefficient amplitude at normal incidence $(\theta=0)$ from DNS (solid line) and theory (dashed line) at acoustic Reynolds number $R e=100$, for coatings of porosity $\phi=0.2$ ( $\phi=0.48(-)$, and $\phi=0.8(-)$. ( a $)$ Cavity aspect ratio $A r=0.06 ;(b) A r=0.12 ;(c) A r=0.3$.

Also, as discussed in section IIA, the presence of a resonant acoustic mode strongly affects the calculation of the reflection coefficient, ${ }^{13}$ and the DNS results are not shown for $\phi=0.2$ in figure $2(b)$ and $(c)$ at high frequencies, where these resonances occur. In these geometries, the resonant disturbances may interact with the boundary-layer flow and cause a detrimental tripping effect. The nonlinear simulations with the outer boundary-layer flow performed in section IIID are aimed in part at addressing this issue.

From figure 2, it is clear that the most efficient attenuative coating is obtained for $A r=0.06, \phi=0.8$ (red curves in figure $2(a)$ ), which would requires many deep pores. Such high porosity can be difficult to achieve in practical application because of structural constraints. Also, the stabilizing effect might not be required for such a wide range of frequency. By restricting our attention to a smaller range of acoustic wave 
frequency around the dominant second-mode frequency, similar or even better acoustic absorptive properties could be obtained with less and larger pores (e.g., black curves in figure $2(b)$ around $f=0.7$ ). The stability of hypersonic boundary layers over UAC operating approximately in a local cancellation/reinforcement regime is investigated in the next section.

Finally, the results also confirmed that a stronger cancellation regime can be obtained for different combinations of porosity and aspect ratio. The existence of a global maximum of acoustic absorption, corresponding to an "optimum" cancellation regime, is presented in the appendix. While current studies on UAC (including the one in this paper) aim at "robust" uniform designs which stabilize a range of frequencies, improvement could potentially be obtained with non-uniform coatings that would lock the dominant second-mode instability in that global maximum of acoustic absorption, and therefore always operate in the "optimum" cancellation regime. The preliminary analysis of such "optimum" streamwise-dependent design is also presented in the appendix.

\section{Temporal boundary layer stability}

\section{A. Numerical setup}

In previous work, ${ }^{11}$ a simplified configuration that considers a $2 \mathrm{D}$ temporally evolving boundary layer on an infinite flat plate with UAC was shown to accurately capture the relevant flow physics for laminar flow control. The restriction to two-dimensional cases is relevant here since the second-mode is initially twodimensional. The temporally evolving boundary layer neglects the spatial growth of the boundary layer, and instead diffuses slowly with time. Over short time-scales associated with acoustic energy attenuation in UAC, the laminar boundary layer is essentially frozen, consistent with either a spatial or temporal description of the mean flow field. The results showed that the second-mode instability has a sufficiently slow streamwise growth to allow spatial instability results to be accurately recovered from the temporal ones via the Gaster transformation. ${ }^{19}$

Details on the code algorithm, implementation and validations can be found in Refs. $(11,20,21)$. In the current study, simulations are performed for both the nonlinear (DNS) and linearized (LDNS) twodimensional Navier-Stokes equations. Periodic boundary conditions are used in the streamwise direction, and the nominally laminar boundary layer spreads in time rather than streamwise position. The grid extends up to $5 H$ in the $y$-direction, with a large buffer zone at the top boundary, to avoid spurious reflections. All the numerical simulations are performed on similar stretched Cartesian grid, with clustering of points near the walls. For the configuration with the porous wall, the mesh contains about half a million grid points, with 100 points per cavity depth, and 12 to 60 points across each cavity length, depending on the pore aspect ratio.

The equations are solved for a perfect gas, with constant specific heat capacities, $\gamma=1.4$ and constant Prandtl number $\operatorname{Pr}=0.7$. Here, the Mach number is $M_{e}=U_{e} / a_{e}=6$, and the wall temperature ratio $T_{w}=T_{w}^{*} / T_{e}^{*}=1.4$ is assumed to be uniform and constant. In this preliminary work, we consider constant viscosity $\left(\mu=\mu_{0}=\mu_{e}\right)$ and conductivity. Temperature variation in these properties will be included in future work. Nevertheless, we showed that the results are qualitatively similar to measurements with temperature-dependent properties, and therefore relevant for UAC design.

All the computations are initialized with an error-function profile for the streamwise velocity (i.e., the correct self-similar solution as $M_{e} \rightarrow 0$ ), uniform pressure and use the Crocco-Busemann relation to compute the initial temperature profile for the chosen wall temperature ratio. The nonlinear simulations are advanced in time until the boundary layer thickness reaches $\delta / H=2$. The resulting boundary layer profile is then frozen and used as the mean flow in linear simulations. An acoustic perturbation is added to the flow and the linearized Navier-Stokes equations are solved. The least damped (or most unstable) eigenmode and the corresponding eigenvalue are then determined from the long-time linear response of the boundary layer, and the nondimensionalized temporal growth/damping rate $\sigma=\sigma^{*} \delta / U_{e}$ and frequency $\omega=\omega^{*} \delta / U_{e}$ are computed. Similarly, the nonlinear simuations are restarted with the same added acoustic perturbation, to quantify nonlinear effects.

Finally, the streamwise extent $\Lambda$ of the domain was chosen to approximately correspond to the wavelength of the most unstable second mode (see section IIIB), and to have an integer number of pores $N_{\text {pore }}$ in the domain. While the typical number of pores per wavelength of instability ${ }^{5}$ is suggested to be about 10 to 20 , its value varies from 3 to 66 in the present simulations. In particular, smaller number of pore are of interest to determine whether detrimental effects such as roughness-induced transition mechanisms are present. 


\section{B. Linear stability at minimum of acoustic absorption}

\begin{tabular}{|c|c|c|c|c|c|c|c|c|c|c|}
\hline cases & flat plate & \multicolumn{3}{|c|}{$\mathrm{UAC} A r=0.06$} & \multicolumn{3}{|c|}{ UAC $A r=0.12$} & \multicolumn{3}{|c|}{$\mathrm{UAC} A r=0.3$} \\
\hline$T_{w}$ & 1.4 & \multicolumn{9}{|c|}{1.4} \\
\hline$M_{e}$ & 6 & \multicolumn{9}{|c|}{6} \\
\hline$\delta / H$ & n.a. & \multicolumn{9}{|c|}{2} \\
\hline$R e_{\delta}$ & 24000 & \multicolumn{9}{|c|}{24000} \\
\hline$R e$ & n.a. & \multicolumn{3}{|c|}{50} & \multicolumn{3}{|c|}{100} & \multicolumn{3}{|c|}{250} \\
\hline$\phi$ & n.a. & 0.2 & 0.48 & 0.8 & 0.2 & 0.48 & 0.8 & 0.2 & 0.48 & 0.8 \\
\hline$\Lambda / \delta$ & 2.5 & 2.4 & 2.5 & 2.475 & 2.4 & 2.5 & 2.475 & 2.25 & 2.5 & 2.4375 \\
\hline$N_{\text {pore }}$ & n.a. & 16 & 40 & 66 & 8 & 20 & 33 & 3 & 8 & 13 \\
\hline
\end{tabular}

Table 1. Parameters for the numerical simulations of a temporally-evolving boundary layer over a flat plate and over nine different coatings in the reinforcement regime. (n.a.: not applicable).

For the first configuration, the nonlinear simulations are advanced in time until the boundary layer thickness satisfies $\delta / H=2$. The parameters for the different numerical simulations are summarized in table (1). Under these conditions, all the linear stability calculations in this section are performed at Reynolds number $R e_{\delta}=\rho_{e} U_{e} \delta / \mu_{e}=24000$, which is related to the acoustic Reynolds number $R e=\rho_{w} a_{w} b / \mu_{w}$ by

$$
R e=\frac{\operatorname{Re}_{\delta} A r}{2 \sqrt{T_{w}} M_{e}} \frac{H}{\delta}
$$

The flow field for the boundary layer over a coating of porosity $\phi=0.2$, cavity aspect ratio $A r=0.3$ is shown in figure $3(a)$, and $(b)$, for the normal velocity $v$ and temperature $T$, respectively. This flow field was used as mean flow for the linear simulation (LDNS) of that geometry. Unlike the boundary layer profile over the flat plate, which is only function of the normal direction, the flow field with UAC is two-dimensional, in particular the velocity field near the cavity mouths. The negative and positive normal velocities at the downstream and upstream cavity edges, respectively, indicate weak recirculation inside the pores. In contrast, the temperature is essentially uniform inside the pores, equal to the wall temperature $T=T_{w}$.

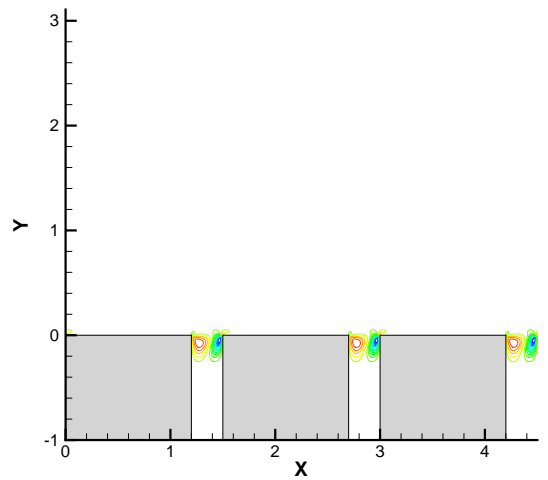

(a)

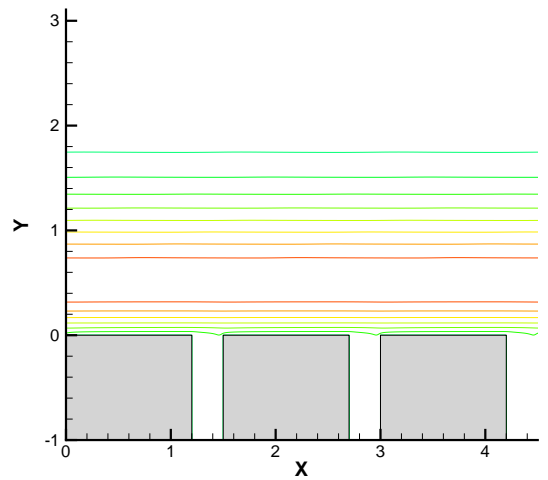

(b)

Figure 3. Flow field from DNS for the hypersonic boundary layer at $M_{e}=6$ over a coating of porosity $\phi=0.2$, cavity aspect ratio $A r=0.3:(a) \mathbf{v} ;(b) \mathbf{T}$. The boundary layer thickness is $\delta / H=2$.

The linear stability theory ${ }^{5}$ (LST) is applied to the two-dimensional second-mode waves at $R e_{\delta}=24000$. These linear calculations are conducted with temperature-independent viscosity for the different mean boundary layer profiles extracted from the DNS of the flat plate and the nine UAC configurations. The growth/decay rate and frequency of the least damped (or most unstable) mode are computed as a function of the streamwise wavelength $\lambda / \delta$. 

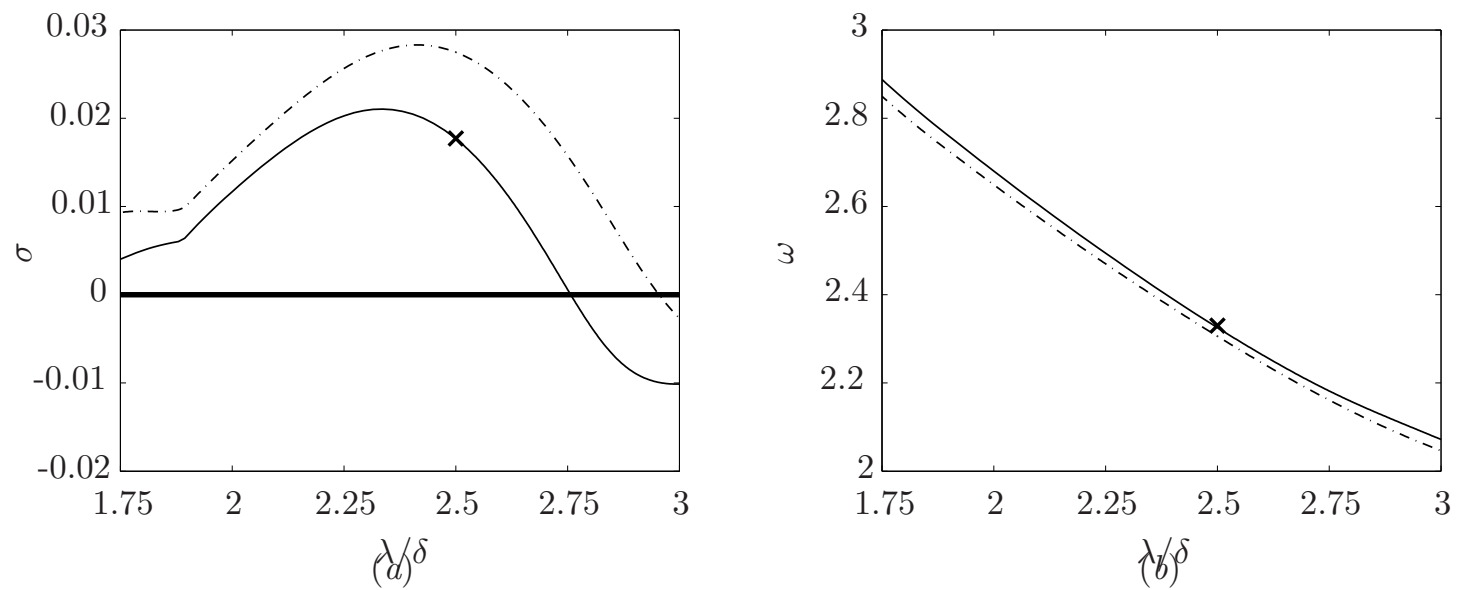

Figure 4. Second-mode instability for a hypersonic boundary layer over a flat plate at $M_{e}=6, R e_{\delta}=24000$ ( - LST, $\times$ LDNS), and $R e_{\delta}=31200$ ( - LST): (a) growth rate; $(b)$ frequency.

The linear results for the hypersonic boundary layer over a flat plate at $R e_{\delta}=24000$ are presented in figure 4. The second-mode had a positive growth rate for streamwise wavelength $1.6 \leq \lambda / \delta \leq 2.75$, and the most unstable mode is at $\lambda / \delta=2.35$, leading to the choice of $\Lambda / \delta \approx 2.5$ for the streamwise extent of the computational domain. The LDNS results match the linear calculation very well, with less than $0.2 \%$ error. The typical second-mode structures are shown in figure $5(a)$, and $(b)$, for the normal velocity $v^{\prime}$ and temperature $T^{\prime}$, respectively. Similarly to the results in Ref. 12, the maximum perturbation is near the wall for the temperature, and near $y=0.3$ for the normal velocity.

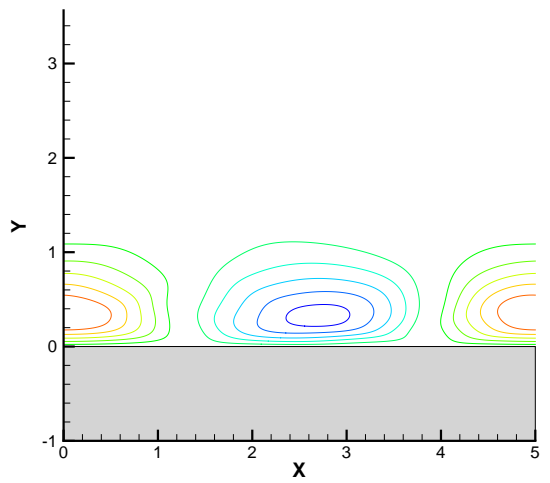

(a)

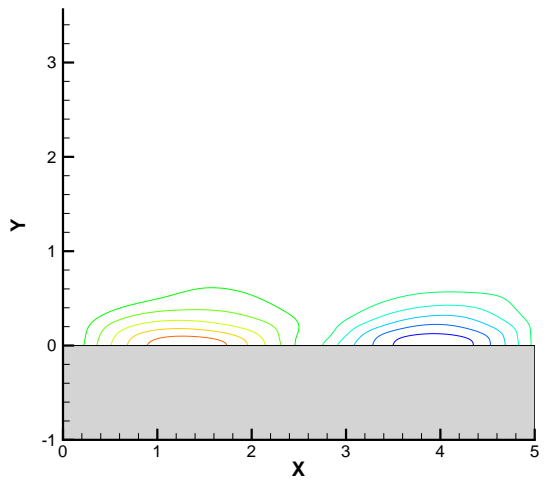

(b)

Figure 5. Structures of the second-mode instability for the hypersonic boundary layer over a flat plate at $M_{e}=6, \operatorname{Re}_{\delta}=24000$ (from LDNS): $(a) v^{\prime} ;(b) T^{\prime}$

The acoustic properties of the different UAC, for the flow conditions and configurations in table (1), are presented in figure 6 . The range of unstable frequencies and the most unstable second-mode for the hypersonic boundary layer over the flat plate $R e_{\delta}=24000$ are also shown in the figure. Here, the frequency of the dominant second-mode is $\omega=\omega^{*} \delta / U_{e}=2.325$, which can be expressed as a function of the acoustic frequency $f=f^{*} H / a_{w}$ using

$$
f=\frac{\omega}{2 \pi} \frac{M_{e}}{\sqrt{T_{w}}} \frac{H}{\delta} .
$$




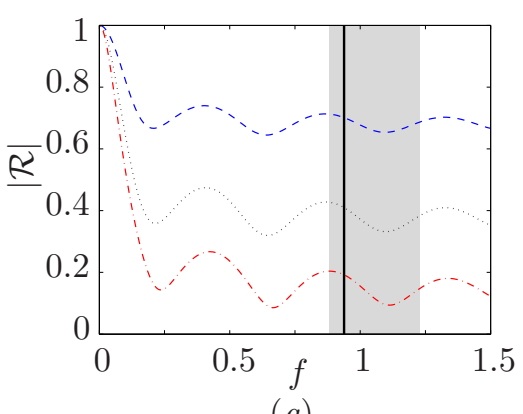

(a)

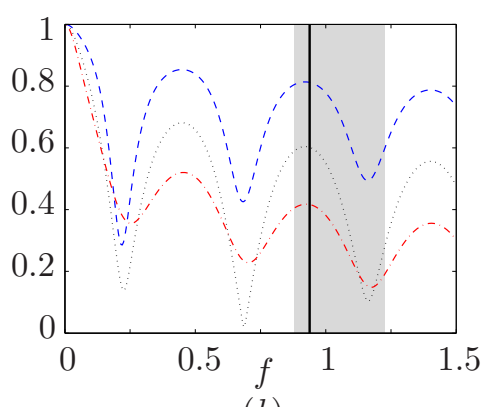

(b)

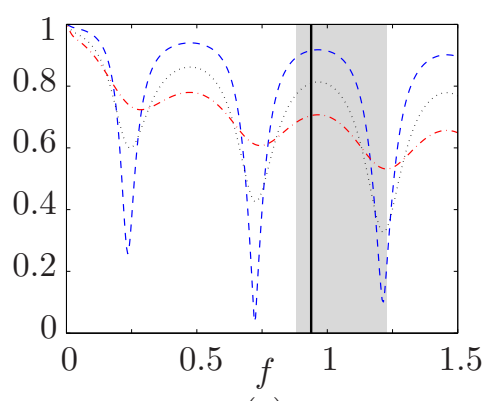

(c)

Figure 6. Reflection coefficient amplitude at normal incidence $(\theta=0)$ from theory, for coatings of porosity $\phi=0.8(--), \phi=0.48(\cdots \cdots)$, and $\phi=0.2(----)$ in table (1). The grey shaded area corresponds to the frequencies of the unstable modes from figure $4(b)$, and the black vertical line is the frequency of the most unstable mode. (a) Cavity aspect ratio $A r=0.06$ at acoustic Reynolds number $\operatorname{Re}=50 ;(b) A r=0.12$ at $R e=100 ;$ (c) $A r=0.3$ at $R e=250$.

As shown in figure 6, the second-mode frequency is approximately at a maximum of the reflection coefficient for all the configurations. These conditions were chosen to quantify the different UAC performance in the reinforcement regime (i.e., the "worst-case scenario"). Since the operating conditions of a uniform $\mathrm{UAC}$ are expected to depend on the streamwise position and alternate between local minima and maxima of the coating acoustic absorption, it is important to make sure the stabilizing effect is obtained even for unfavorable conditions.

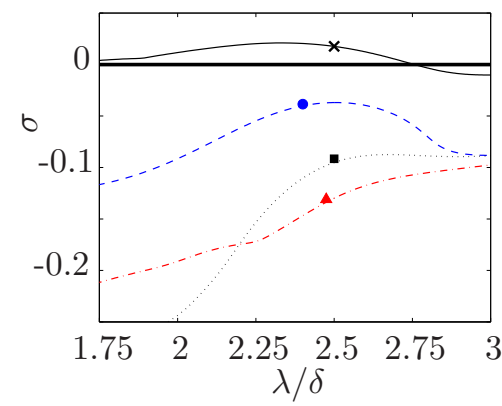

$(a)$

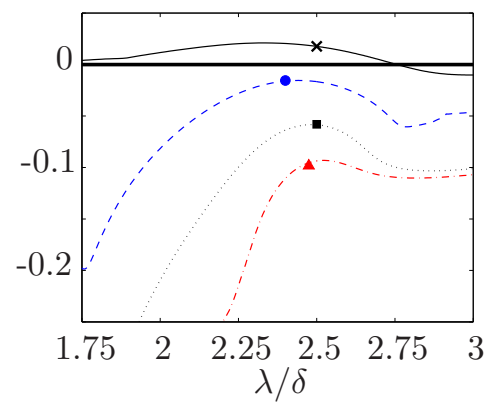

(b)

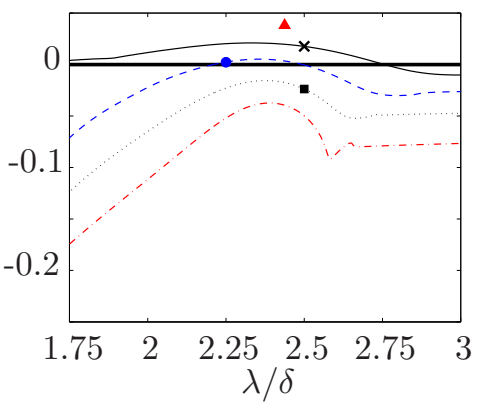

$(c)$

Figure 7. Linear growth rate $\sigma$ of the second-mode instability for a hypersonic boundary layer at $M_{e}=6$ and $R e_{\delta}=24000$ over a flat plate $(-$ LST, $\times$ LDNS $)$ and over coatings of porosity $\phi=0.2(----\quad$ LST; $\bullet$ LDNS), $\phi=0.48$ ( …... LST; LDNS), and $\phi=0.8$ ( - — LST; $\triangle$ LDNS ). (a) Cavity aspect ratio $A r=0.06$; (b) $A r=0.12 ;(c) A r=0.3$.

The results for the linear growth rate are compared to the LDNS estimates in figure $7(a),(b)$ and $(c)$, for the UAC of aspect ratios $A r=0.06, A r=0.12$ and $A r=0.3$, respectively. There are again a very good agreement between the LST and the LDNS, with less than $5 \%$ error on the growth rate. Similar agreement is obtained for the mode frequency. Even in this configuration corresponding to a minimum of the coating acoustic absorption, almost all the UAC have negative growth rate and stabilize the second-mode instability, demonstrating again the robustness of the method. The exception is the case of a coating of large pores and low porosity $(A r=0.3, \phi=0.2$ in figure $7(c))$ which still exhibits a small positive growth rate, both in the LST and LDNS. As expected, the very low number of pores per wavelength of instability $\left(N_{\text {pores }}=3\right)$ and the poor acoustic scattering performance $(|\mathcal{R}|>0.9$ at the second mode frequency) of this particular design lead to a less efficient coating. The second-mode structures for the boundary layer over this geometry is presented in figure 8 , similar to the results for the flat plate in figure 4 .

The numerical simulations also identify the coating of aspect ratio $A r=0.3$, and porosity $\phi=0.8$ as a special case. Here, an unstable mode is observed in the long-time linear response of the perturbed flow over 


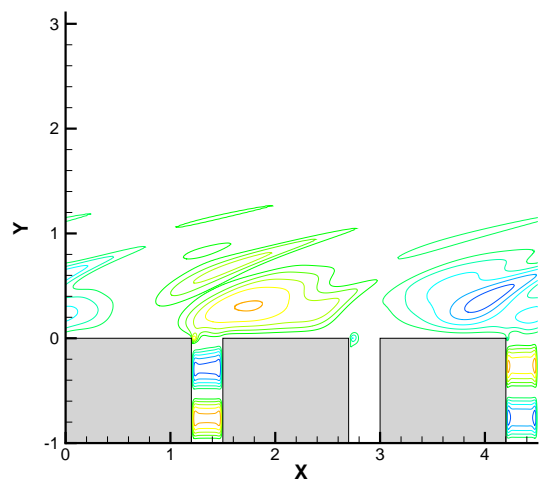

(a)

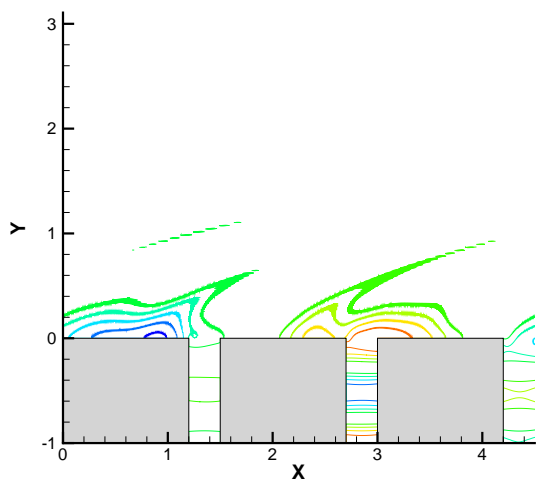

(b)

Figure 8. Structure of the second-mode instability for the hypersonic boundary layer over a coating of porosity $\phi=0.2$ and cavity aspect ratio $A r=0.3$, at $M_{e}=6, R e_{\delta}=24000$ (from LDNS): (a) v'; (b) T'

the UAC (red triangle in figure $7(c)$ ). The preliminary results on this mode are discussed in section IIID. Further analysis is underway to identify the origin of the instability.

\section{Linear stability at maximum of acoustic absorption}

For the second configuration, the nonlinear simulations are continued until the boundary layer thickness satisfies $\delta / H=2.6$, and the linear stability calculations are now performed at Reynolds number $R e_{\delta}=31200$. The LST results for the hypersonic boundary layer over the flat plate is compared to the previous case in figure 4. As expected, the growth rate of the second-mode has increased, leading to a wider range of unstable streamwise wavelength. The change in frequency is not significant. The most unstable mode has now a frequency $w=2.361$, and a wavelength $\lambda / \delta=2.41$.

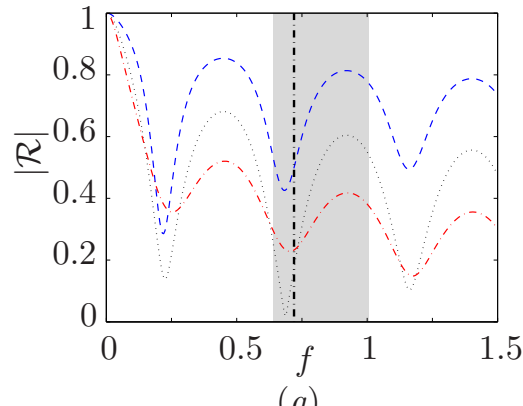

(a)

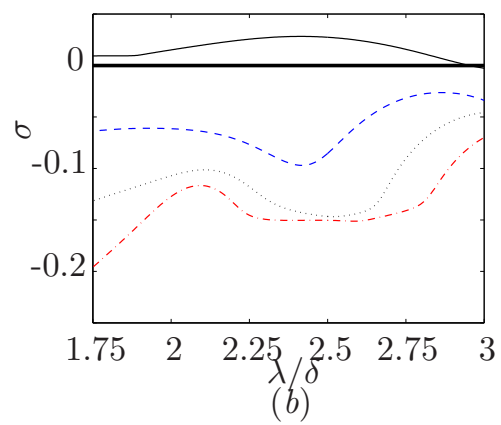

(b)

Figure 9. Improvement of the performance of coatings of aspect ratio $A r=0.12$ and porosity $\phi=0.2(----)$,

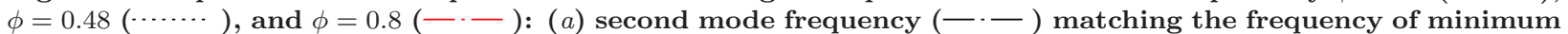
reflection coefficient amplitude. The frequencies of the unstable modes (grey shaded area) are also presented; (b) corresponding linear growth rate from LST at $R e_{\delta}=31200$ for the UAC and the flat plate ( $\left.~-~\right)$.

In contrast, the acoustic Reynolds number and the acoustic scattering properties of the coating are unchanged. Using equation (3), the frequency of the most unstable second-mode now approximately corresponds to a local minimum of the reflection coefficient, as shown in figure $9(a)$. These conditions correspond to a cancellation regime (i.e., "best-case scenario"), where the UAC operates at a local maximum of acoustic absorption. The corresponding the linear growth rates are presented in figure 9(b). As anticipated, a significant stabilizing effect is obtained for this configuration, over a large range of wavelength, compared to the results in figure $7(b)$. Numerical simulations are underway to confirm these results. 


\section{Nonlinear simulations}

Nonlinear numerical simulations including the outer flow are performed for the UAC configurations in the "reinforcement" regime, to confirm the linear results and investigate potential detrimental effects of the coating. For each of the cases considered in table (1), the nonlinear simulations agreed fully with the results of linear analysis. That is, except for the particular case of $\phi=0.8$ and $A r=0.3$ discussed below, the nonlinear simulations all resulted in damped second mode response and the boundary layer remained laminar.

As mentioned in section IIIB, an unstable mode is observed in the linear response of the perturbed boundary layer over a coating of aspect ratio $A r=0.3$, and porosity $\phi=0.8$. The same instability growth is present in the nonlinear simulations. The current DNS are being extended to longer times to investigate whether the mode eventually reaches an asymptotic state, limit cycle or continues to evolve into more complicated fluctuations. The frequency and growth rate for this unstable mode can be estimated at $\omega=0.74$ and $\sigma=0.0382$, which means that this configuration exhibits a dominant instability of growth rate larger than that of the most unstable second mode. Also the instability wavelength is half of the streamwise extent of the domain (i.e., approximately half of the most unstable wavelength of the second mode). These values do not correspond to the second mode, nor the acoustic resonant mode discussed in the section IIA. Additional work is underway for this geometry to identify the nature of this instability and investigate its destabilizing effect of the porous coating.

Also, as discussed in section IIA, a resonant acoustic mode of frequency $f_{\text {res }}=\phi / A r$ was observed in the DNS (without outer flow) during the investigation of the coating acoustic properties. Here, the term "resonant" is used to qualify the mode frequencies in a broad sense, even though the oscillations are actually lightly damped and decay in time. Except for coating of low porosity, these frequencies are higher than the ultrasonic frequency band relevant for UAC, and are not expected to impact the coating absorptive performance. To confirm this assumption, additional spectral analysis of the flow field for the different coatings of porosity $\phi=0.2$ is performed for the nonlinear runs. In most cases, small peak in the spectrum around the frequency of the acoustic mode are observed. However, these resonant acoustic modes were all stable and eventually died out without tripping the boundary layer. Therefore, the results of the nonlinear simulations with the outer flow indicate that the coupling of these small-scale scattered waves generated by neighboring pores do not destabilize the second-mode.

Finally, it should be mentioned that the present study of roughness-induced transition mechanisms has several limiting assumptions. First, only strictly two-dimensional, planar cavities are considered. Second, the periodic domain was fixed to approximately one wavelength of the dominant second mode. Thus, any first mode instability would not be captured, even if they were unstable, since their wavelength is longer than what is permitted in the computational domain. Third, the present simulations were performed with constant (temperature-independent) properties. While this assumption is not expected to significantly alter our current results, the temperature effects need to be included in future work. With these limitations in mind, the nonlinear results did not show differences with the linearized simulations. The DNS did not show evidence of roughness-induced transition mechanisms, even for small numbers of pores per wavelength of instability (as low as 3 pores).

\section{Conclusions}

The stabilizing effect of ultrasonic absorptive coatings on hypersonic boundary layer instabilities is investigated using direct numerical simulations of the linear and nonlinear two-dimensional Navier-Stokes equations. Following our previous studies, temporally (rather than spatially) evolving boundary layers are considered, as this approximation was shown to be appropriate to slowly-growing second-mode instabilities.

The coatings consist of a uniform array of rectangular pores (slots) of aspect ratios $A r=0.06,0.12,0.3$, and porosity $\phi=0.2,0.48,0.8$. The conservative way to design coatings is to have the UAC operated in an attenuative regime where the pores are relatively deep and acoustic waves (and second mode instabilities) are attenuated by viscous effects inside the pores. Here, alternate designs are investigated, where the pores are relatively shallow and the coating operates in an alternating cancellation/reinforcement regime, depending on the frequency of the acoustic waves.

The second-mode properties for a hypersonic boundary layer at Mach number $M_{e}=6$ and Reynolds number $R e_{\delta}=24000$ over a flat plate and over the different UAC are computed using linear stability theory and linearized direct numerical simulations. These configurations correspond to the reinforcement regime 
where the second-mode frequency matches a frequency of minimum absorptive properties of the coating (i.e., "worst case scenario"). It is important to consider these cases to ensure that the UAC stabilizing effect is obtained even in unfavorable conditions. For all but one of the cases considered, the linear DNS confirm the results of linear instability theory that employs an approximate porous-wall boundary condition. For these cases, damping rates and wavelengths agree precisely with linear theory.

The particular case of a coating $\phi=0.8$ and $A r=0.3$ showed the existence of a shorter wavelength instability that is not predicted by theory. For that geometry, the instability is present in both linear and nonlinear simulations, with a growth rate larger than that of the most unstable second mode. The origin and effects of this instability will be investigated in future work.

Finally, nonlinear simulations of the same cases were performed and led to the same conclusions than the linear analysis. The porous coatings damp second mode instability and the boundary layer remains laminar. Additionally, the nonlinear results did not exhibit any "tripping" of the boundary layer by small scale disturbances associated with individual pores. Overall, the presence of the porous coating did not seem to have any significant detrimental effect (aside from the new instability at $A r=0.3, \phi=0.8$ under investigation). In future work, the temperature-dependent properties will be implemented. To continue the study of roughness-induced transition mechanisms, the computational domain will be extended in the streamwise direction to allow for the (potential) development of the first mode instability.

\section{Acknowledgments}

This work is supported by the Air Force of Scientific Research under Grant FA9550-08-1-0251 managed by Dr. John Schmisseur, and partially (Fedorov) by the AFOSR/NASA National Center for Hypersonic Research in Laminar-Turbulent Transition. Computer time was provided in part by the Department of Defense High Performance Computing centers.

\section{Appendix}

This appendix presents the analysis to optimize UAC design based on the acoustic scattering properties of the porous coating without external boundary-layer flow, and on the characteristics of the second mode without the coating. Since the theoretical model described in Refs. $(14,15)$ was validated by the numerical simulations over the range of parameters relevant to UAC in hypersonic flight, it will be the main tool for the optimization of the coating properties

First, the model is used to find the optimum value(s) of porosity, cavity aspect ratio and acoustic Reynolds number that lead to a global minimum (or local minima) of the reflection coefficient amplitude. Then, within this set of parameters, the appropriate values of the UAC parameters (i.e., mainly the cavity depth) are identified, such that the frequency of minimum reflection matches the frequency of the most amplified second-mode for an hypersonic boundary layer over a flat plate.

For the particular UAC designs considered here, the range of frequency for the second-mode instability was estimated from several experiments at $0.1<f<0.35$. Consequently, all the following analysis is performed using the theoretical reflection coefficient $\mathcal{R}=\mathcal{R}(\phi, A r, R e, f)$ computed as a function of porosity $(0.2 \leq \phi \leq 0.8)$, cavity aspect ratio $(0.05 \leq A r \leq 0.3)$, and Reynolds number $(50 \leq R e \leq 1000)$, for incident acoustic wave of frequency $0.15 \leq f \leq 0.35$ at normal incidence $\left(\theta=0^{\circ}\right)$.

\section{Details on critical Reynolds number}

The critical Reynolds number $R e_{\text {crit }}$ for the presence of a local minimum of the reflection coefficient around $f \approx 0.25$, is characterized as a function of porosity and aspect ratio within the range mentioned in the previous section. As expected, $R e_{\text {crit }}$ is related to viscous and thermal absorption inside the pores and is only a function of $A r$. From the theoretical modeling, the dependence of the critical Reynolds number on the aspect ratio is $R e_{\text {crit }} \approx 2 / A r$. Similar estimates were obtained using the theory of the propagation of acoustic waves in narrow and wide tubes ${ }^{22}$

Now, this critical Reynolds numbers is compared to the range of acoustics Reynolds numbers relevant for practical UAC in high-altitude hypersonic flight. Recall that the Mach number and temperature ratio 
are defined as

$$
M_{e}=\frac{U_{e}}{a_{e}}, \quad T=\frac{T^{*}}{T_{e}^{*}}=\left(\frac{a}{a_{e}}\right)^{2} .
$$

From the ideal gas law and the power law for viscosity $\left(\mu / \mu_{r}=\left(T^{*} / T_{r}^{*}\right)^{n}\right.$, where the subscript $r$ denotes the reference quantity, here the boundary-layer edge property), the ratio of the kinematic viscosities satisfies

$$
\frac{\nu}{\nu_{e}}=\left(\frac{T^{*}}{T_{e}^{*}}\right)^{n+1}=T^{n+1} .
$$

Also, an expression for the nondimensionalized stagnation temperature $T_{0}$ can be derived from the compressible boundary layer energy equation:

$$
T_{0}=1+\frac{\gamma-1}{2} M_{e}^{2} .
$$
as

Using equations (4), (5), and (6), the unit acoustic Reynolds number at the wall $R e_{w}^{*}$ can be expressed

$$
R e_{w}^{*}=R e_{0}^{*}\left(\frac{1+\frac{\gamma-1}{2} M_{e}^{2}}{T_{w}}\right)^{n+1 / 2}
$$

Here, the unit ambient acoustic Reynolds number $R e_{0}^{*}=a_{0} / \nu_{0}$ is computed as a function of altitude (see figure 10(a)), using the properties of the 1976 standard atmosphere, and the power law for viscosity, with the reference quantity corresponding to the ambient properties at sea level and $n=0.7$.

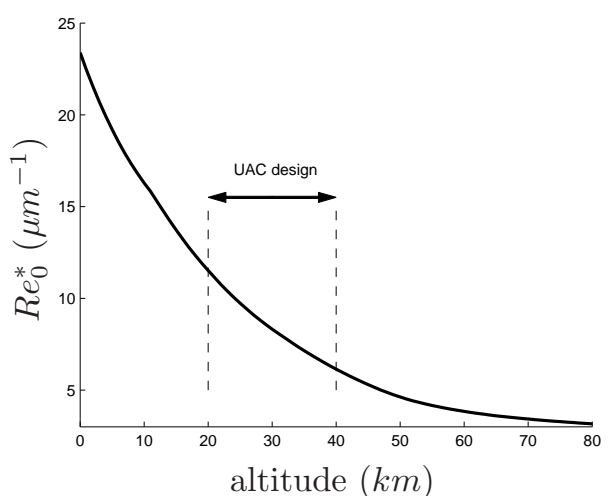

(a)

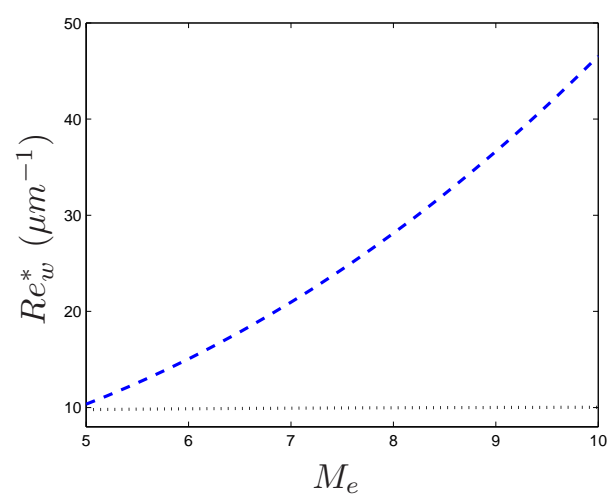

(b)

Figure 10. (a) Unit ambient acoustic Reynolds number as a function of altitude; (b) unit acoustic Reynolds number at the wall as a function of Mach number, for flight at $30 \mathrm{~km}$ altitude; Wall temperature ratio $T_{w}=5$ $(----)$, and $T_{w}=T_{a d}(\cdots \cdots)$.

The parameter $R e_{w}^{*}$ is shown in figure $10(b)$ as a function of Mach number, for a given design altitude $(30 \mathrm{~km})$ and different wall temperature ratio. Therefore, the acoustic Reynolds number is $R e=b R e_{w}^{*}$, and for pores of dimension $b>10 \mu \mathrm{m}$, is larger than its critical value, $R e>R e_{\text {crit }}$, for most flight conditions and cavity aspect ratio. Since the range of cavity half-width considered for UAC is typically $10 \mu m<b<100 \mu m$, we anticipate the presence of reflection from the cavity bottom, and therefore, local minimum of the reflection coefficient in most cases, making possible the optimization process described in this appendix.

\section{Optimization of the coating acoustic absorption}

First, from the theoretical formulation of the reflection coefficient in Ref 14, we notice that the dependence on Reynolds number and aspect ratio can be described by the lumped parameter $\operatorname{ArRe}$, so that $\mathcal{R}=$ $\mathcal{R}(\phi, A r R e, f)$. Then, for a given porosity and lumped parameter, the reflection coefficient amplitude is minimized with respect to frequency (see figure 11). Finally, the parametric dependence of this minimum 


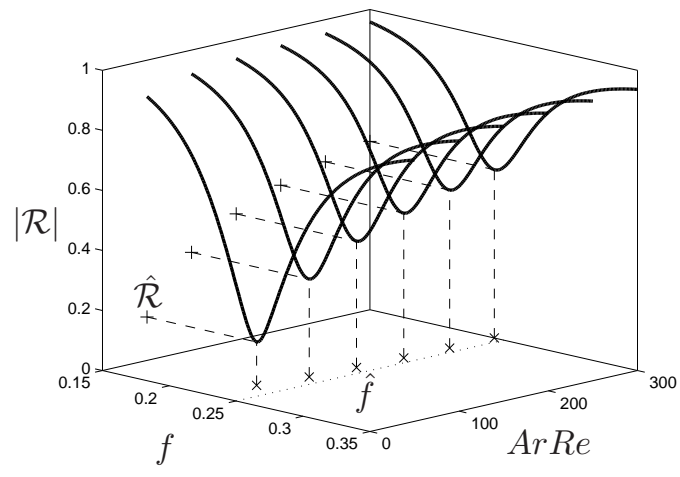

(a)

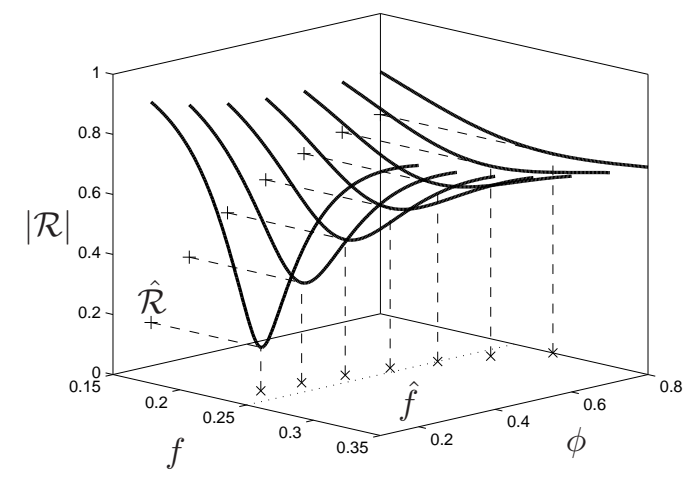

(b)

Figure 11. Reflection coefficient amplitude as a function of frequency, for constant porosity $\phi=0.2$ in $(a)$, and constant $\operatorname{ArRe}=50$ in $(b)$. The computed minima $\hat{\mathcal{R}}=\min _{f}|\mathcal{R}|(+)$ and corresponding frequency $\hat{f}(\times)$ are also shown.

$\hat{\mathcal{R}}(\phi, A r R e)=\min _{f}|\mathcal{R}|$ and of the corresponding frequency $\hat{f}(\phi, A r R e)$ are tabulated, as shown in figure 12 (a) and (b), respectively.

From these figures, it is clear that, for a given porosity, there is an optimum value of the lumped parameter $A r R e_{\text {opt }}$ such that $\hat{\mathcal{R}}\left(\phi, A r R e_{\text {opt }}\right) \approx 0$. These values are computed by minimizing $\hat{\mathcal{R}}$ with respect to $\operatorname{Ar} R e$, and the resulting reflection coefficient amplitude $\min _{A r R e} \hat{\mathcal{R}}$ and corresponding frequency $\min _{\operatorname{ArRe}} \hat{f}$ are shown in figure $13(a)$ as a function of porosity. Likewise, for a given $A r R e$, there is an optimum porosity $\phi_{\text {opt }}$, but in that case, the minimum reflection coefficient amplitude $\min _{\phi} \hat{\mathcal{R}}$ is not uniformly zero for the range of parameter considered here (see figure $13(b)$ ). That is, for large Reynolds number or large aspect ratio, such that $A r R e>30$, the optimum coating cannot cancel the incoming acoustic wave completely.

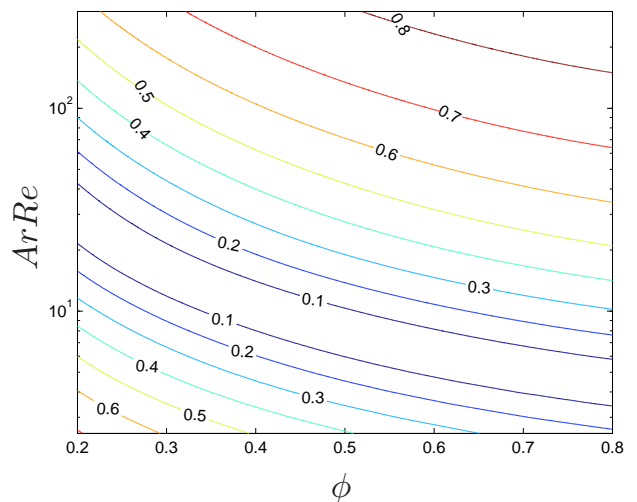

(a)

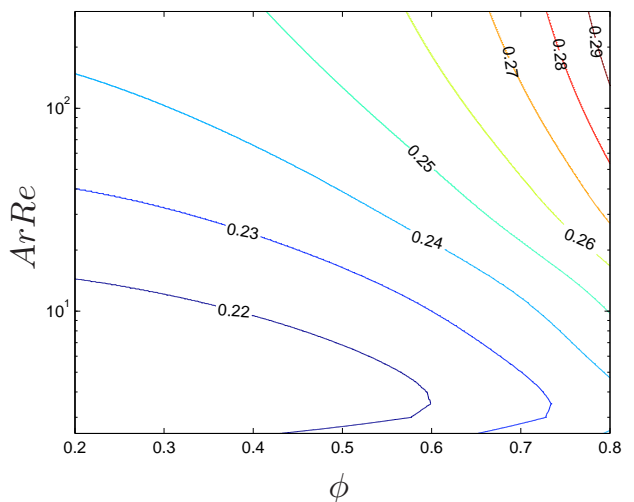

(b)

Figure 12. Contours of the minimum of reflection coefficient amplitude $\hat{\mathcal{R}}$ in $(a)$ and corresponding frequency $\hat{f}$ in $(b)$, as a function of the porosity $\phi$ and lumped parameter $\operatorname{ArRe}$ (log scale).

From these figures, it is clear that, for a given porosity, there is an optimum value of the lumped parameter $A r R e_{\text {opt }}$ such that $\hat{\mathcal{R}}\left(\phi, A r R e_{\text {opt }}\right) \approx 0$. These values are computed by minimizing $\hat{\mathcal{R}}$ with respect to $\operatorname{Ar} R e$, and the resulting reflection coefficient amplitude $\min _{A r R e} \hat{\mathcal{R}}$ and corresponding frequency $\min _{\operatorname{Ar} R e} \hat{f}$ are shown in figure $13(a)$ as a function of porosity. Likewise, for a given $A r R e$, there is an optimum porosity $\phi_{o p t}$, but in that case, the minimum reflection coefficient amplitude $\min _{\phi} \hat{\mathcal{R}}$ is not uniformly zero for the range of parameter considered here (see figure $13(b)$ ). That is, for large Reynolds number or large aspect ratio, such that $A r R e>30$, the optimum coating cannot cancel the incoming acoustic wave completely. 
Using a least-squares fitting method on the data from the top and bottom figures in 13 (a), we obtained the following approximated relationship between the porosity and the lumped parameter for zero reflection coefficient, and the corresponding frequency:

$$
\begin{gathered}
\operatorname{ArRe}=A \phi^{-\beta} \approx 3.05 \phi^{-1.41}, \\
f_{a c s}=\min _{A r R e} \hat{f} \approx 0.13 \phi^{2}-0.10 \phi+0.24 .
\end{gathered}
$$

Here, we notice that the frequency of minimum reflection is only a weak function of the porosity. To further show that the method is robust, that is, a coating with parameters satisfying equation (8) would still be effective for acoustic waves of frequency slightly different than $f_{a c s}$, the reflection coefficient amplitude at frequency $f_{a c s} \pm 10 \%$ error is computed as a function of porosity and lumper parameter. Even with $10 \%$ error on the frequency, the reflection coefficient amplitude of the optimum coating remains less than 0.3 . As a result, we will assume that the frequency of minimum reflection is independent of the porosity and lumped parameter, and approximate $f_{a c s}$ to its mean and its standard deviation, i.e., $f_{a c s} \approx 0.225 \pm 0.005$.

Therefore, for incoming acoustic waves of frequency $f_{a c s}$ (normal incidence), the reflection coefficient is zero for a coating such that the porosity $\phi$, cavity aspect ratio $A r$, and acoustic Reynolds number Re satisfy equation (8). That is, we can design different porous coatings that would completely absorbed acoustic waves of frequency $f_{a c s}$.

\section{Optimization of the second mode damping}

The previous results is now formulated in terms of the properties of the second mode (or Mack mode) of instability for an hypersonic boundary layer over a flat plate. The goal here is to relate the frequency of minimum acoustic reflection $f_{a c s}$ to the second-mode angular frequency $\omega_{2 n d}$, which is typically expressed as: $^{23}$

$$
\omega_{2 n d}=2 \pi f^{*} \frac{\delta_{B l}}{U_{e}}=F \sqrt{R e_{x}},
$$

where $\delta_{B l}=\sqrt{\nu_{e} x / U_{e}}$ is the Blasius length scale, $F=2 \pi f^{*} \nu_{e} / U_{e}^{2}$ is the nondimensionalized frequency, and $R e_{x}=U_{e} x / \nu_{e}$ is the Reynolds number based on the streamwise coordinates $x$ and edge velocity $U_{e}$.

Here, we assume that the frequency $\omega_{2 n d}$ of the local most unstable second mode is approximately constant, independent of the streamwise location. Starting with the nondimensionalized frequency $f$ from the acoustic scattering analysis, we have

$$
f=\frac{f^{*} H}{a_{w}}=\frac{f^{*} \nu_{e}}{U_{e}^{2}} \frac{U_{e} x}{\nu_{e}} \frac{U_{e}}{a_{e}} \frac{a_{e}}{a_{w}} \frac{H}{x}=\frac{1}{2 \pi} F \operatorname{Re}_{x} M_{e} \frac{1}{\sqrt{T_{w}}} \frac{H}{x} .
$$

where the subscripts $e$ and $w$ denotes the boundary-layer edge and wall conditions, respectively.

Matching the frequency of minimum reflection $\omega_{a c s}=2 \pi f_{a c s}$ from the acoustic scattering analysis, with the second-mode frequency $\omega_{2 n d}=F \sqrt{R e_{x}}$, and assuming these frequencies to be approximately constant, we obtain that the cavity depth satisfies

$$
\frac{H}{x} \sqrt{R e_{x}}=\frac{\omega_{a c s}}{\omega_{2 n d}} \frac{\sqrt{T_{w}}}{M_{e}} .
$$

While coatings currently used in application are typically uniform, this analysis shows that the optimum pore depth increases in the streamwise direction like $\sqrt{x}$. The dependence arises from the fact that the second-mode properties depend on the streamwise location, that is, at each $x$-station, we are dealing with several unstable waves of slightly different frequencies. ${ }^{5}$ By enforcing this streamwise-dependent depth, the coating always operates at the maximum absorption of the frequency of the local most unstable second mode.

The equation (12) can be written to express the cavity depth directly as $H=H(x)$. Introducing the ambient stagnation properties (subscript 0), we have

$$
\frac{H}{x} \sqrt{R e_{x}}=\frac{H}{\sqrt{x}} \sqrt{\frac{U_{e}}{\nu_{e}}}=\frac{H}{\sqrt{x}} \sqrt{\frac{U_{e}}{a_{e}} \frac{a_{e}}{a_{0}} \frac{a_{0}}{\nu_{0}} \frac{\nu_{0}}{\nu_{e}}} .
$$

Using (5) and the unit ambient acoustic Reynolds number $R e_{0}^{*}=a_{0} / \nu_{0}$ (see figure $10(a)$ ), equation (13) becomes

$$
\frac{H}{x} \sqrt{R e_{x}}=\frac{H}{\sqrt{x}} \sqrt{M_{e} \frac{1}{\sqrt{T_{0}}} R e_{0}^{*} T_{0}^{n+1}} .
$$



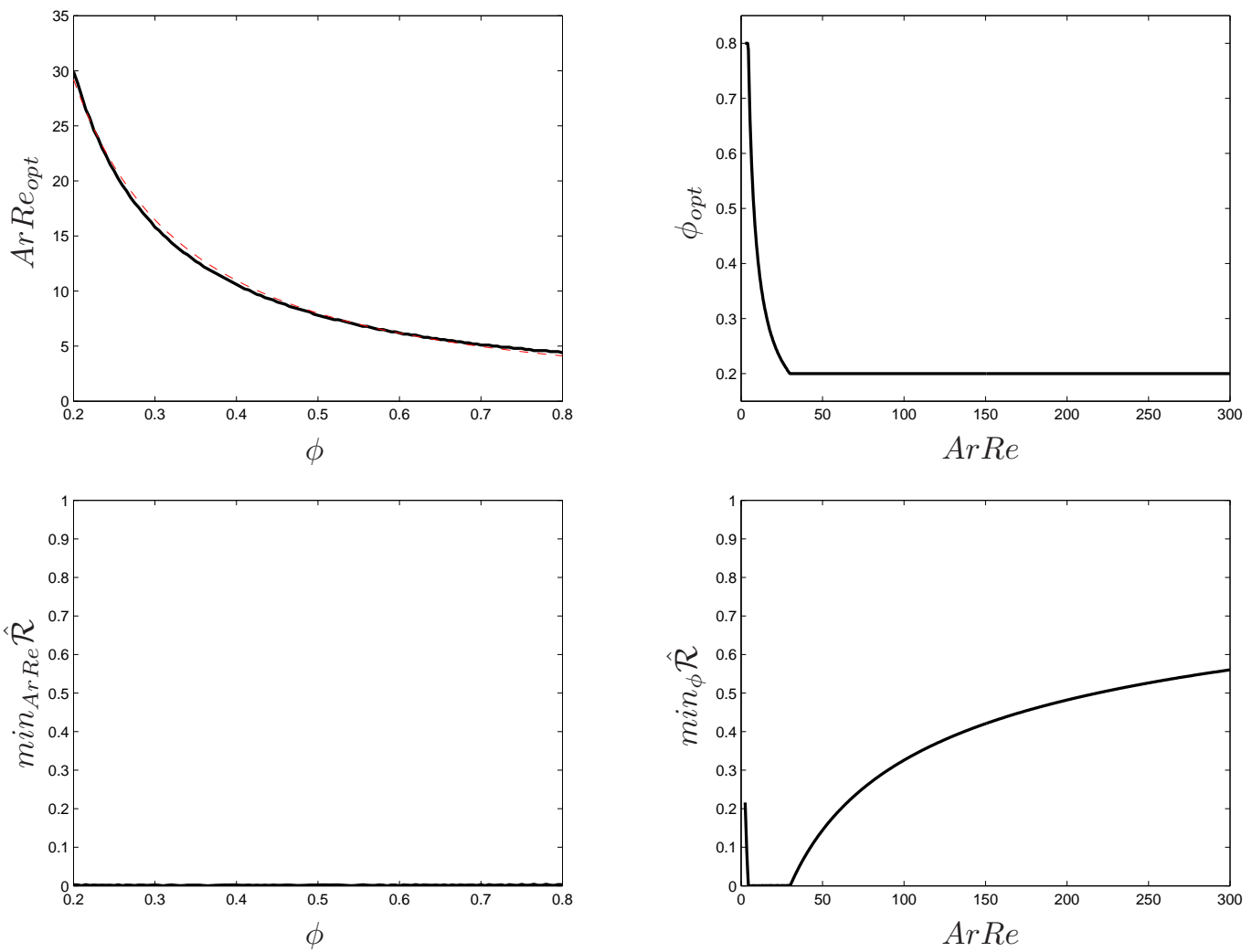

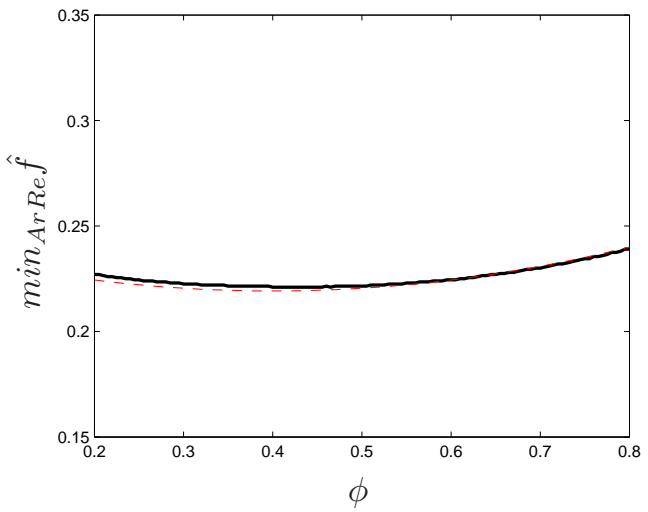

(a)

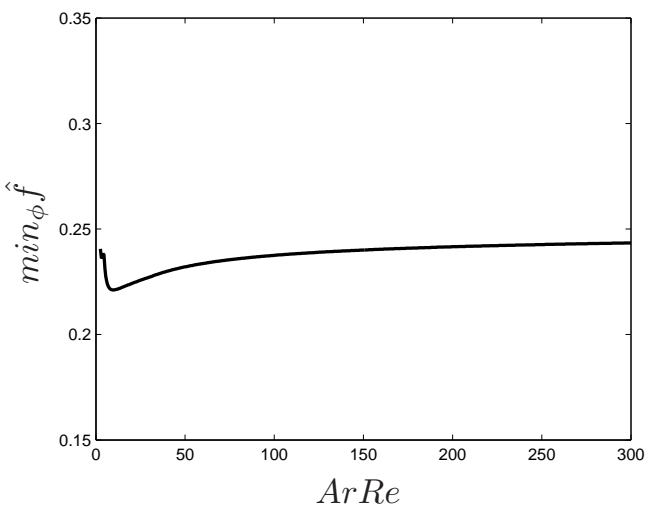

(b)

Figure 13. Minimization of the reflection coefficient amplitude from figure 12 with respect to $A r R e(a)$, and with respect to $\phi(b)$; from top to bottom: optimum parameter, minimum of reflection coefficient amplitude, and corresponding frequency. ( ---- ) fitted curve from equations (8) and (9). 
Combining equations (12), (14), and (6), the optimum pore depth is given by

$$
H=\frac{\omega_{a c s}}{\omega_{2 n d}} \frac{\sqrt{T_{w}}}{M_{e}^{3 / 2}\left(1+\frac{\gamma-1}{2} M_{e}^{2}\right)^{n / 2+1 / 4}} \frac{1}{\sqrt{R e_{0}^{*}}} \sqrt{x}
$$

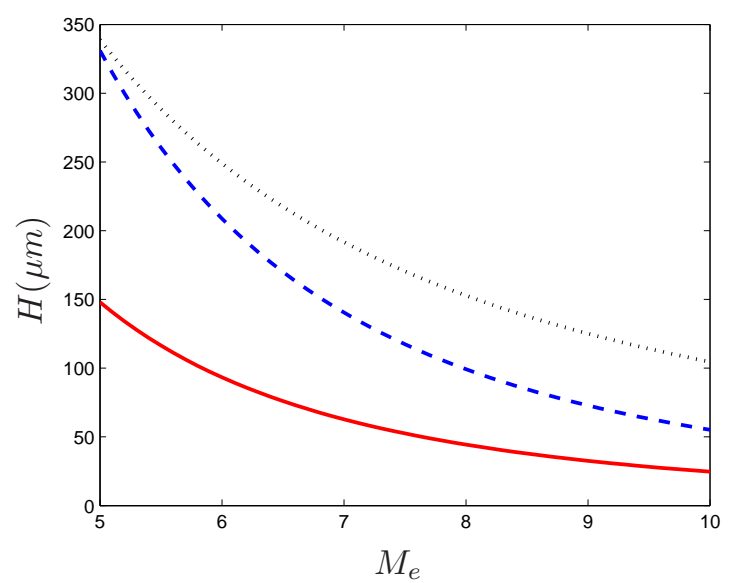

Figure 14. Optimum pore depth at $x=1 \mathrm{~m}$ for flight at $30 \mathrm{~km}$ altitude with second-mode frequency $w_{2 n d}=0.1$; Wall temperature ratio $T_{w}=1(-), T_{w}=5(---)$, and $T_{w}=T_{a d}(\cdots \cdots)$.

The optimum pore depth at $x=1 \mathrm{~m}$ is shown in figure 14 at a given design altitude, for different wall temperature ratio. Here, we used a typical value of the second mode frequency $w_{2 n d}=0.1, f_{a c s}=0.225$ from the previous analysis, and $n=0.7$. The choice of $T_{w}=1$ corresponds to cold-wall conditions relevant to experiments in shock tunnel. For practical applications, the wall temperature ranges from $T_{w} \approx 5$ for moderate cooling, to $T_{w}=T_{a d}$, where $T_{a d}=T_{a d}^{*} / T_{e}^{*} \approx 1+\sqrt{\operatorname{Pr}}(\gamma-1) M_{e}^{2} / 2$ is the adiabatic temperature ratio.

\section{Example of optimum design with constant porosity}

Here, our analysis shows that the optimum pore depth is smaller than the typical value used for uniform coating (e.g., $H \approx 500 \mu m$ ) and would lead to thinner coating easier to fabricate. For a given vehicle design speed and wall temperature, equations (8), (15) and figure 10(a) can therefore be used to design several different configurations of "optimum" ultrasonic absorptive coating. For instance, an optimum UAC with constant low porosity could be design in the following fashion:

1. Choose the vehicle design speed (e.g., $M_{e}=7$ ), wall temperature (e.g., $T_{w}=T_{a d}$ ) and altitude (e.g., $30 \mathrm{~km}$ ). Determine the unit acoustic Reynold number at the wall using figure $10(a)$ and equation $(7)$.

2. Compute the optimum pore depth $H(x)$ using equation (15).

3. Choose the porosity. Here, we fix $\phi=0.2$

4. Use equations (8) and (7) to find the optimum cavity half-width $b(x)$

5. Finally, the optimum spacing is given by $s(x)=2 b(x) / \phi$

The coating parameters $H, b$ and $s$ are shown in figure 15 as a function of the streamwise location $x$ on the vehicle. This design correspond to a coating with low porosity and large cavity aspect ratio, that is, a thinner coating with less pores, therefore simpler to manufacture.

\section{References} 1990.

${ }^{1}$ Malik, M. R., Zang, T. A., and Bushnell, D. M., "Boundary layer transition in hypersonic flows," AIAA Paper 90-5232, 


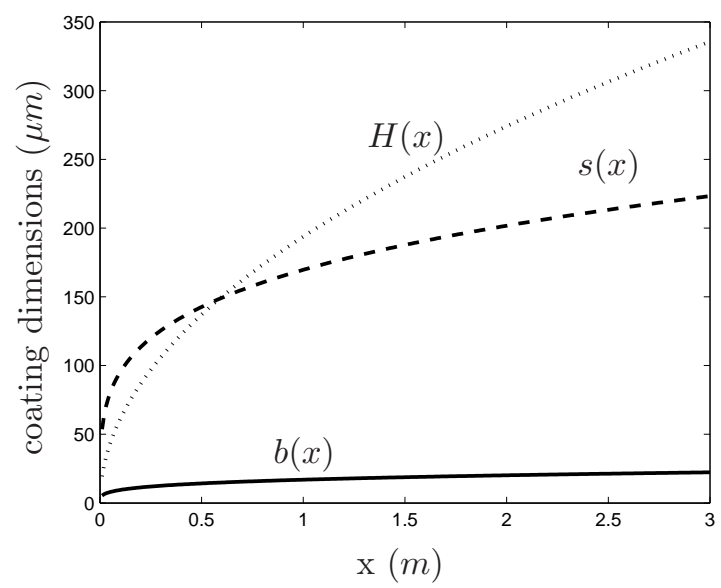

Figure 15. Optimum design of coating with constant porosity $\phi=0.2$, for flight at $30 \mathrm{~km}$ altitude, $M_{e}=7$ and $T_{w}=T_{a d}$ : pore spacing $s(---)$, pore depth $H(\cdots \cdots)$ ) and half-width $b\left(-\frac{1}{-}\right.$.

${ }^{2}$ Schneider, S., "Flight Data for Boundary Layer Transition at Hypersonic and Supersonic Speeds," J. Spacecraft and Rockets, Vol. 36(1), 1999, pp. 8-20.

${ }^{3}$ Schneider, S., "Hypersonic Laminar-Turbulent Transition on Circular Cones and Scramjet Forebodies," Progress in Aerospace Sciences, Vol. 40(1-2), 2004, pp. 1-50.

${ }^{4}$ Kimmel, R., "Aspects of hypersonic boundary layer transistion control," AIAA Paper 2003-0772, 2003.

${ }^{5}$ Fedorov, A. V., Malmuth, N. D., Rasheed, A., and Hornung, H. G., "Stabilization of hypersonic boundary layers by porous coatings," AIAA J., Vol. 39(4), 2001, pp. 605-610.

${ }^{6}$ Rasheed, A., Hornung, H. G., Fedorov, A. V., and Malmuth, N. D., "Experiments on passive hypervelocity boundary layer control using an ultrasonically absorptive surface," AIAA J., Vol. 40(3), 2002, pp. 481-489.

${ }^{7}$ Fedorov, A. V., Shiplyuk, A. N., Maslov, A. A., Burov, E. V., and Malmuth, N. D., "Stabilization of a hypersonic boundary layer using and ultrasonically absorptive coating," J. Fluid Mech., Vol. 479, 2003, pp. 99-124.

${ }^{8}$ Fedorov, A. V., Kozlov, V. F., Shiplyuk, A. N., Maslov, A. A., Sidorenko, A. A., Burov, E. V., and Malmuth, N. D., "Stability of hypersonic boundary layer on porous wall with regular microstructure," AIAA Paper 2003-4147, 2003.

${ }^{9}$ Bountin, D., Shiplyuk, A., Maslov, A., and Chokani, N., "Nonlinear aspects of hypersonic boundary layer stability on a porous surface," AIAA Paper 2004-0255, 2004.

${ }^{10}$ Maslov, A. A., Shiplyuk, A. N., Sidorenko, A. A., Polivanov, P., Fedorov, A. V., Kozlov, V. F., and Malmuth, N. D., "Hypersonic laminar flow control using a porous coating of random microstructure," AIAA Paper 2006-1112, 2006.

${ }^{11}$ Brès, G. A., Colonius, T., and Fedorov, A. V., "Stability of temporally evolving supersonic boundary layers over microcavities for ultrasonic absorptive coatings," AIAA Paper 2008-4337, 2008.

${ }^{12}$ Sandham, N. D. and Lüdeke, H., "A numerical study of Mach 6 Boundary layer stabilization by means of a porous surface," AIAA Paper 2009-1288, 2009.

${ }^{13}$ Brès, G. A., Colonius, T., and Fedorov, A. V., "Interaction of acoustic disturbances with micro-cavities for ultrasonic absorptive coatings," AIAA Paper 2008-3903, 2008.

${ }^{14}$ Brès, G. A., Colonius, T., and Fedorov, A. V., "Acoustic properties of Porous Coatings for Hypersonic Boundary Layer Control," AIAA J., to be published, 2009.

${ }^{15}$ Kozlov, V. F., Fedorov, A. V., and Malmuth, N. D., "Acoustic properties of rarefied gases insides pores of simple geometries," J. Acoust. Soc. Amer., Vol. 117(6), 2005, pp. 3402-3412.

${ }^{16}$ Stetson, K. F., Thomson, E. R., Donaldson, J. C., and Siler, L. G., "Laminar boundary-layer stability experiments on a cone at Mach 8, part 1: sharp cone," AIAA Paper 83-1761, 1983.

${ }^{17}$ Egorov, I. V., Fedorov, A. V., Novikov, A. V., and Soudakov, V. G., "Direct numerical simulation of supersonic boundarylayer stabilization by porous coatings," AIAA Paper 2007-948, 2007.

${ }^{18}$ Malmuth, N. D., Fedorov, A. V., Shalaev, V., Cole, J., Khokhlov, A., Hites, M., and Williams, D., "Problems in high speed flow prediction relevant to control," AIAA Paper 98-2695, 1998.

${ }^{19}$ Gaster, M., "A note on the relation between temporally-increasing and spatially-increasing disturbances in hydrodynamic stability," J. Fluid Mech., Vol. 14, 1962, pp. 222-224.

${ }^{20}$ Colonius, T. and Lele, S., "Computational Aeroacoustics: Progress in Nonlinear Problems of Sound Generation," Progress in Aerospace Sciences, Vol. 40, No. 6, 2004, pp. 345-416.

${ }^{21}$ Brès, G. A. and Colonius, T., "Three-dimensional instabilities in compressible flow over open cavities," J. Fluid Mech., Vol. 599, 2008, pp. 309-339.

${ }^{22}$ Pierce, A. D., Acoustics, an introduction to its physical principles and applications, McGraw-Hill, 1989.

${ }^{23}$ Mack, L. M., "Linear stability theory and the problem of supersonic boundary-layer transition," AIAA J., Vol. 13, 1975, pp. $278-289$. 